\title{
Prolonged response to trabectedin in a patient with ovarian carcinosarcoma refractory to adjuvant platinum-taxane
}

\author{
Edgar Petru, Vassiliki Kolovetsiou, Tina I dris \\ Department of Obstetrics and Gynecology, Medical University of Graz, Graz, Austria \\ Correspondence: Edgar Petru. Address: Department of Obstetrics and Gynecology, Medical University of Graz, \\ Auenbruggerplatz 14, A-8036 Graz, Austria. E-mail: edgar.petru@medunigraz.at
}

Received: February 15, 2013

Accepted: March 19, 2013

DOI : $10.5430 /$ jst.v3n3p9

URL: http://dx.doi.org/10.5430/jst.v3n3p9

Online Published: March 24, 2013

\begin{abstract}
Background: Carcinosarcoma of the ovary carries an unfavorable prognosis and the optimal chemotherapeutic regimen for this disease is yet to be determined. To the authors' knowledge, this is the first report of a metastatic ovarian carcinosarcoma successfully treated with trabectedin monotherapy after failure of platinum/taxane-based therapy.

Case report: The case of a platinum-refractory advanced ovarian carcinosarcoma in a 73-year-old female is presented. After initial tumor debulking, the patient received adjuvant carboplatin and paclitaxel. After 2 cycles, tumor progression occurred. The authors speculated that rapid tumor progression occurred as a consequence of regrowth of sarcomatous elements within the carcinosarcoma. This platinum-refractory disease was treated with single-agent trabectedin $1.5 \mathrm{mg} / \mathrm{m}^{2}$ every three weeks. A prolonged partial response for 13 months along with an acceptable safety profile was achieved. During this time, the patient was able to perform her daily activities without restrictions.

Conclusion: Our case suggests that trabectedin monotherapy may represent a viable therapeutic option for patients with platinum-refractory advanced ovarian carcinosarcoma.
\end{abstract}

\section{Key words}

Trabectedin, Ovarian carcinosarcoma, Muellerian mixed tumor, Chemotherapy, Ovary

\section{Introduction}

Ovarian carcinosarcoma, also known as malignant mixed Müllerian or mesodermal tumor (MMMT), is uncommon accounting for approximately $1 \%$ to $2 \%$ of all ovarian malignancies ${ }^{[1,2]}$. In the female genital tract, carcinosarcomas usually arise in the uterus and much less frequently in the ovary, vagina and cervix, with a tendency to affect the elderly population ${ }^{[3]}$. Carcinosarcomas are particularly aggressive neoplasms histologically characterized by the presence of epithelial carcinomatous elements and a stromal sarcomatous homologous or heterologous component.

Ovarian carcinosarcomas carry a particularly unfavorable prognosis. No effective chemotherapeutic regimen and radiotherapy exist. Optimal cytoreductive surgical debulking is crucial and the FIGO stage is considered as the only prognostic factor ${ }^{[2]}$. 
Herein a case of a platinum-refractory ovarian carcinosarcoma in a patient who experienced disease progression during adjuvant chemotherapy with carboplatin and paclitaxel is reported. The authors assumed that rapid tumor progression was caused by the regrowth of sarcomatous elements within the carcinosarcoma. In addition, trabectedin has shown activity in

platinum-resistant cells in vitro ${ }^{[4]}$. On this rational basis, the patient was treated with trabectedin. The rarity of carcinosarcoma of the ovary and a prolonged response to trabectedin monotherapy prompted this report.

\section{Case report}

In October 2010, a 73 year-old woman presented with abdominal cramps and vomiting. She had a history of hypertension and non-traumatic thrombosis. Ultrasound revealed an $18 \mathrm{~cm}$ solid-multicystic mass in the left adnexal region, significant ascites, and signs of peritoneal carcinomatosis. Computed tomography (CT) scan showed non-enlarged retroperitoneal lymph nodes. In November 2010, the patient underwent total abdominal hysterectomy, bilateral salpingo-oophorectomy, omentectomy, appendectomy and systematic pelvic and paraaortic lymphadenectomy. Lymph nodes were negative and no macroscopic tumor residuals were left at surgery. Histological analysis revealed a primary ovarian carcinosarcoma of FIGO stage IIIc showing a predominant component of clear cell undifferentiated carcinoma with a stromal component containing chondroid differentiation.

Postoperatively, the patient received carboplatin AUC 6 and paclitaxel $175 \mathrm{mg} / \mathrm{m}^{2}$ every three weeks. After two cycles, platinum-refractory disease was diagnosed with significant deterioration of the patient's performance status (Karnofsky 70). Subileus symptoms, upper abdominal pain, recurrent vomiting and nausea were present. Gynecologic examination revealed a left pelvic lesion of $2-3 \mathrm{~cm}$ in diameter. Abdominopelvic CT scan demonstrated a new $2 \mathrm{~cm}$ pelvic lesion, signs of peritoneal carcinomatosis with lesions up to $1 \mathrm{~cm}$ in diameter, and abundant ascites. Serum CA-125 was $61 \mathrm{U} / \mathrm{mL}$. After ascitic drainage of 2,200 mL, tumor progression was confirmed cytologically.

In January 2011, the patient received her first cycle of trabectedin at $1.5 \mathrm{mg} / \mathrm{m}^{2}$ administered through a central venous line as 24-hour infusion every three weeks. After two cycles, thrombosis of the right deep femoral vein occurred and therapeutic doses of dalteparin were given. After the fourth cycle of trabectedin, the tumor showed a partial remission with no palpable pelvic lesion at gynecologic examination. At that time, her CT scan revealed no evidence of ascites and showed a significant decrease of peritoneal carcinomatosis. Additionally, the level of CA-125 decreased to $21 \mathrm{U} / \mathrm{mL}$. The patient received four additional cycles of trabectedin until July 2011. The most severe adverse events were grade 2 herpes zoster and a urinary tract infection, whilst the most common laboratory abnormalities were grade 2 neutropenia and grade 1 anemia. No signs of liver toxicity were observed. One year after the initiation of trabectedin monotherapy the patient was alive with no radiological or clinical evidence of disease. The patient had a Karnofsky status of 90 and was able to perform her daily activities without restrictions. Thirteen months after the initiation of trabectedin, the tumor progressed intraperitoneally and the patient died three months thereafter.

\section{Discussion}

To the authors' knowledge, this is the first report of a metastatic ovarian carcinosarcoma treated with trabectedin monotherapy after failure of platinum and a taxane. This case illustrates a potential role of trabectedin which may reinforce the therapeutic armamentarium in ovarian carcinosarcoma after failure of front-line therapy. After eight cycles of trabectedin, a durable response was observed. The latter was accompanied by significant palliation of symptoms, and with acceptable toxicity. Although non-cumulative myelosuppression, with reversible neutropenia as the predominant component, and transient transaminase elevation are well-known as the most common laboratory abnormalities seen with trabectedin, in the present case no such events have been observed ${ }^{[5,6]}$.

This case is not the first that describes a patient with carcinosarcoma of the ovary minimally responding to chemotherapy used for epithelial carcinomas but reaching a complete response after receiving chemotherapy used for sarcomas ${ }^{[7]}$. 
Trabectedin is approved for the treatment of patients with advanced soft tissue sarcoma after failure of anthracyclines and ifosfamide as well as for the treatment patients with platinum-sensitive ovarian cancer in conjunction with pegylated liposomal doxorubicin ${ }^{[8]}$. Thus, trabectedin is one of few drugs being active against both ovarian cancer and soft tissue sarcoma.

Owing to the rarity of ovarian carcinosarcomas, there is no established chemotherapy regimen ${ }^{[2]}$. Lesier et al have evaluated the combination of platinum and taxane either after initial tumor resection or as neoadjuvant therapy ${ }^{[9]}$. Between 1991 and 2005, 30 patients with ovarian carcinosarcoma were identified for analysis, underscoring the rarity of these tumors and the difficulty of enrolment of patients into prospective clinical trials ${ }^{[1,9,10]}$.

In conclusion, in a 73 year-old woman with ovarian carcinosarcoma, refractory to adjuvant platinum and taxane treatment, trabectedin induced a prolonged response with an acceptable toxicity profile.

\section{References}

[1] Muller M, Dupre PF, Lucas B, Simon H, Malhaire JP, Guillemet C, et al. [Carcinosarcoma of the ovary]. Journal de gynecologie, obstetrique et biologie de la reproduction. 2007; 36(4): 399-402. PMid:17408876 http://dx.doi.org/10.1016/j.jgyn.2007.03.001

[2] Brown E, Stewart M, Rye T, Al-Nafussi A, Williams AR, Bradburn M, et al. Carcinosarcoma of the ovary: 19 years of prospective data from a single center. Cancer. 2004; 100: 2148-53. PMid:15139057 http://dx.doi.org/10.1002/cncr.20256

[3] O'Toole RV, Tuttle SE, Shah NT. Heterologous carcinosarcoma of the fallopian tube. A case report. J Reproduct Med. 1982; 27: 749-52. PMid:7161756

[4] Soares DG, Machado M, Rocca C, Poindessous V, Quaret D et al. Trabectedin and its C subunit modified analogue PM01183 attenuate nucleotide excision repair and show activity toward platinum-resistant cells. Mol Cancer Ther. 2011; 10: 1481-1489. PMid:21622731 http://dx.doi.org/10.1158/1535-7163.MCT-11-0252

[5] Carter NJ, Keam SJ. Trabectedin: a review of its use in soft tissue sarcoma and ovarian cancer. Drugs. 2010; 70: 355-76. PMid:20166769 http://dx.doi.org/10.2165/11202860-000000000-00000

[6] Monk BJ, Herzog TJ, Kaye SB, Krasner CN, Vermorken JB, Muggia FM, et al. Trabectedin Plus Pegylated Liposomal Doxorubicin in Recurrent Ovarian Cancer. J Clin Oncol. 2010; 28: 3107-14. PMid:20516432 http://dx.doi.org/10.1200/JCO.2009.25.4037

[7] Demetri G, Chawla S, Von Mehren M, Ritch P, Baker L, Blay J, Hande K et al. Efficacy and safety of trabectedin in patients with advanced or metastatic liposarcoma or leiomyosarcoma after failure of prior anthracyclines and ifosfamide: Results of a randomized phase II study of two different schedules. J Clin Oncol. 2009; 27(25): 4188-4196. PMid:19652065 http://dx.doi.org/10.1200/JCO.2008.21.0088

[8] Simon SR, Wang SE, Uhl M, Shackney S. Complete response of carcinosarcoma of the ovary to therapy with doxorubicin, ifosfamide, and dacarbazine. Gynecol Oncol. 1991; 41: 161-6. http://dx.doi.org/10.1016/0090-8258(91)90278-D

[9] Leiser AL, Chi DS, Ishill NM, Tew WP. Carcinosarcoma of the ovary treated with platinum and taxane: the memorial Sloan-Kettering Cancer Center experience. Gynecol Oncol. 2007; 105: 657-61. PMid:17395252 http://dx.doi.org/10.1016/j.ygyno.2007.01.037

[10] Thigpen JT, Blessing JA, Olt G, Lentz SS, Bell J. Cisplatin as second-line therapy in ovarian carcinoma treated initially with single-agent paclitaxel: a Gynecologic Oncology Group study. Gynecol Oncol. 2003; 90: 581-6. http://dx.doi.org/10.1016/S0090-8258(03)00327-5 